\section{EFEITO DA DIETA HIPERLIPÍDICA E DO TREINAMENTO AERÓBICO NA ATEROSCLEROSE EM CAMUNDONGOS apoE-/-}

EFFECT OF HYPERLIPIDIC DIET AND AEROBIC TRAINING ON ATHEROSCLEROSIS IN apoE-/- MICE

EFECTO DE LA DIETA HIP
EN RATONES apoE-/-

Silvio Anderson Toledo Fernandes (Educador Físico) ${ }^{1}$

Antônio José Natali

(Educador Físico) $^{2}$

Sérgio Luis Pinto da Matta

$(\text { Biólogo })^{3}$

Bruno Gonzaga Teodoro

(Educador Físico) ${ }^{1}$

Frederico Souzalima Caldoncelli

Franco (Educador Físico) ${ }^{4}$

Mateus Camaroti Laterza

(Educador Físico) $^{5}$

Maria do Carmo Gouveia Peluzio

(Nutricionista) $^{6}$

1. Núcleo de Educação Física, Instituto Federal do Sudeste de Minas Gerais - Juiz de Fora, MG, Brasil.

2. Departamento de Educação Física, Universidade Federal de Viçosa - Viçosa, MG, Brasil. 3. Departamento de Biologia Geral, Universidade Federal de Viçosa - Viçosa, MG, Brasil. 4. Departamento de Educação Física, Instituto Federal do Sudeste de Minas Gerais - Rio Pomba, MG, Brasil.

5. Faculdade de Educação Física e Desportos Universidade Federal de Juiz de Fora - Juiz de Fora, MG, Brasil.

6. Departamento de Nutrição e Saúde - Viçosa, MG, Brasil.

\section{Correspondência:}

Rua Bernardo Mascarenhas, 1.327/302B, 36080-001 - Juiz de Fora, MG, Brasil.

silvio.fernandes@ifsudestemg.edu.br

\section{RESUMO}

Introdução: A população brasileira tem seguido a tendência dos países desenvolvidos, cuja característica é o aumento do consumo de gorduras e sedentarismo. Objetivo: Investigar os efeitos da dieta hiperlipídica e do treinamento sobre o peso corporal, o consumo alimentar, a massa corporal magra, os lipídios plasmáticos, o peso do fígado e a aterosclerose em camundongos com deficiência de apolipoproteína E (apoE-/-). Métodos: vinte e seis camundongos foram divididos em grupos/dieta: NS (normolipídica e sedentário), HS (hiperlipídica e sedentário), NT (normolipídica e treinamento) e HT (hiperlipídica e treinamento). Aferiu-se peso corporal (PC), consumo alimentar (CA) e peso relativo do fígado (PRF). Calculou-se a água da carcaça (A\%) pela diferença do peso pré e pós-secagem, gordura (G\%) por Soxhlet e proteína (P\%) por Kjeldahl. Determinaram-se colesterol total (CT), triglicerídeos (TG) e HDL por método enzimático-colorimétrico e LDL e VLDL pela formula de Friedewald. Analisou-se a aterosclerose por fotomicrografia. Utilizou-se ANOVA e o método de Duncan, com $P<0,05$. Resultados: No $P C$ inicial e final, não houve diferença de $G \%$ e $P \%$ entre os grupos $(P>0,05)$. A dieta hiperlipídica aumentou o CA (365,3 $\pm 5,09$ vs. 340,16 $\pm 6,32)$ e LDL (452,20 $\pm 114,63$ vs. $329,60 \pm 77,54)$ nos animais sedentários e o CT $(858,75 \pm 140,84$ vs. 320,87 $\pm 157,81)$ e o LDL $(839,36 \pm 139,94$ vs. $282,66 \pm 166,92)$ nos treinados ( $\mathrm{P}<0,05)$. Já o treinamento aumentou o CA $(350,4 \pm 7,81$ vs. $340,16 \pm 6,32)$ e $A \%(64,23 \pm 2,46$ vs. 62,62 $\pm 1,47)$ nos camundongos normolipídicos e diminuiu o CA (336,68 $\pm 6,23$ vs. 365,3 $\pm 5,09)$, TG $(22,75 \pm 12,36$ vs. $66,00 \pm 21,88), \operatorname{HDL}(14,83 \pm 3,95$ vs. $37,60 \pm 13,12), \operatorname{VLDL}(4,55 \pm 2,47$ vs. $13,20 \pm 4,37)$ e aterosclerose $(0,35 \pm$ $0,13$ vs. $0,56 \pm 0,12)$ nos hiperlipídicos $(P<0,05)$. Conclusão: A dieta hiperlipídica pode desencadear distúrbios no metabolismo lipídico e, com isso, desencadear doenças cardiovasculares; porém, associada ao treinamento, pode diminuir a aterosclerose.

Palavras-chave: metabolismo, estilo de vida sedentário, composição corporal.

\section{ABSTRACT}

Introduction: The Brazilian population has followed the trend of developed countries, whose characteristic is the increased consumption of fats and sedentary lifestyle. Objective: To investigate the effects of high fat diet and training on body weight, food intake, lean body mass, plasma lipids, liver weight and atherosclerosis in mice with apolipoprotein E (apoE---) deficiency. Methods: 26 rats were divided into groups/diet: NS (normolipidic and sedentary), HS (high fat and sedentary), NT (normolipidic and training) and HT (high fat and training). Body weight (BW), food intake (FI) and relative liver weight (RLW) were measured in a Gehaka BG2000 scales. The water from the carcass (A\%) was calculated from the weight difference between the pre-and post-drying, the fat (\% F) was calculated by Soxhlet, and the protein (P\%) by Kjeldahl. We determined total cholesterol (TC), triglycerides (TG) and HDL by colorimetric enzymatic method and VLDL and LDL by the Friedewald formula. Atherosclerosis was analyzed by photomicrography. We used ANOVA as well as the Duncan's method, $P<0.05$. Results: In the initial and final $B W$, there was no difference in $F \%$ and $P \%$ between

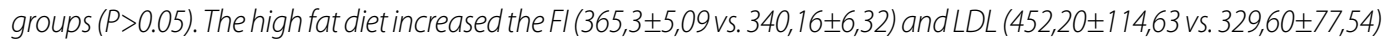
in the sedentary animals and TC $(858,75 \pm 140,84$ vs. $320,87 \pm 157,81)$ and $\operatorname{LDL}(839,36 \pm 139,94$ vs. $282,66 \pm 166,92)$ in trained ones $(P<0.05)$. On the other hand, training increased the $C A(350,4 \pm 7,81$ vs. $340,16 \pm 6,32)$ and $A \%(64,23 \pm 2,46$ vs. 62,62 $\pm 1,47)$ in normolipidic mice and decreased FI $(336,68 \pm 6,23$ vs $365,3 \pm 5,09)$, TG $(22,75 \pm 12,36$ vs. $66,00 \pm 21,88)$, $\operatorname{HDL}(14,83 \pm 3,95$ vs. $37,60 \pm 13,12), V L D L(4,55 \pm 2,47$ vs. $13,20 \pm 4,37)$ and atherosclerosis $(0,35 \pm 0,13$ vs. 0,56 $\pm 0,12)$ in hyperlipidic mice $(P<0.05)$. Conclusion: A high fat diet can trigger disturbances in the lipid metabolism and thereby trigger cardiovascular disease, but when associated with activity may decrease atherosclerosis.

Keywords: metabolism, sedentary lifestyle, body composition.

\section{RESUMEN}

Introducción: La población brasileña ha seguido la tendencia de los países desarrollados, cuya característica es el aumento del consumo de grasas y el sedentarismo. Objetivo: Investigar los efectos de la dieta hiperlipídica y del entrenamiento sobre el peso corporal, el consumo de alimentos, l a masa corporal delgada, los lípidos plasmáticos, el peso del hígado y la aterosclerosis en ratones con deficiencia de apolipoproteína E (apoE--).). Métodos: 26 ratones fueron divididos 
en grupos/dieta: NS (normolipídica y sedentario), HS (hiperlipídica y sedentario), NT (normolipídica y entrenamiento) y HT (hiperlipídica y entrenamiento). Se controlaron peso corporal (PC), consumo de alimentos (CA) y peso relativo del hígado (PRH). Se calculó el agua de la carcasa (A\%) por la diferencia del peso antes y después del secado, grasa (G\%) por Soxhlet y proteína (P\%) por Kjeldahl. Se determinaron colesterol total (CT), triglicéridos (TG) y HDL por el método enzimático-colorimétrico, y LDL y VLDL por la fórmula de Friedewald. Por fotomicrografía se analizó la aterosclerosis. Se utilizó ANOVA y el método de Duncan, con $P<0,05$. Resultados: En el PC inicial y final, no hubo diferencia de G\% y P\%

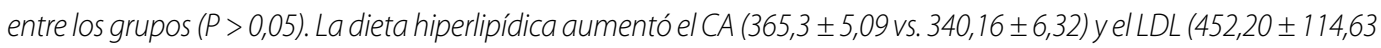
vs. 329,60 $\pm 77,54)$ en los animales sedentarios, y el CT $(858,75 \pm 140,84$ vs. 320,87 $\pm 157,81)$ y el LDL $(839,36 \pm 139,94 \mathrm{Vs}$. $282,66 \pm 166,92)$ en los entrenados $(P<0,05)$. Desde otro punto de vista, el entrenamiento aumentó el $C A(350,4 \pm 7,81$ vs. $340,16 \pm 6,32)$ y $A \%(64,23 \pm 2,46$ vs. 62,62 $\pm 1,47)$ en los ratones normolipídicos y disminuyó el CA $(336,68 \pm 6,23$ vs. $365,3 \pm 5,09), T G(22,75 \pm 12,36$ vs. $66,00 \pm 21,88), \operatorname{HDL}(14,83 \pm 3,95$ vs. $37,60 \pm 13,12), V L D L(4,55 \pm 2,47$ vs. 13,20 $\pm 4,37)$ y la aterosclerosis $(0,35 \pm 0,13$ vs. 0,56 $\pm 0,12)$ en los hiperlipídicos $(P<0,05)$. Conclusión: La dieta hiperlipídica puede causar disturbios en el metabolismo lipídico y, con eso, producir enfermedades cardiovasculares; no obstante, si está asociada con el entrenamiento, puede reducir la aterosclerosis.

Palabras clave: metabolismo, estilo de vida sedentario, composición corporal.

Artigo recebido em 28/12/2010, aprovado em 04/10/2013.

\section{INTRODUÇÃO}

A dieta da população ocidental é caracterizada por ser rica em calorias e hiperlipídica. Nos Estados Unidos a ingestão calórica total proveniente de lipídios fica em torno de 36\%; no Canadá, esta proporção se eleva para $42 \%{ }^{1}$ e, no Brasil, esta realidade também tem-se mostrado presente ${ }^{2}$. Estudos relatam que, independente da origem étnica, indivíduos que consomem grande quantidade de gordura, principalmente do tipo saturada, têm níveis elevados de colesterol sérico e maior incidência de aterosclerose em relação àqueles com menor consumo de gordura ${ }^{3}$.

Além de adotar uma dieta com baixo consumo de gordura, a prática regular de atividade física está, também, associada à menor predisposição aos fatores de risco para doenças cardiovasculares 4 . Porém, nem todos esses resultados encontrados são confirmados em indivíduos com alterações genéticas, especificamente, no gene que expressa a apoliproteína $E$ (apoE). Tem-se demonstrado que indivíduos deficientes em apoE, ou até mesmo com polimorfismo dessa apoliproteína, podem desenvolver aterosclerose ${ }^{5,6}$. A apoE é uma proteína integrante de todas as lipoproteínas. Essa apolipoproteína tem sido extensivamente estudada, principalmente por sua função no metabolismo dos lipídios e pelo envolvimento no transporte de colesterol em vários tecidos ${ }^{6}$.

O metabolismo lipídico pode ser influenciado por vários fatores. Dentre eles, estão o fato de que indivíduos treinados oxidam mais ácidos graxos e que uma dieta rica em lipídeos aumenta a oxidação destes. Sendo esses fatores decorrentes de adaptações enzimáticas que, se associados, podem estar atuando de maneira cumulativa ${ }^{7}$. Nessa perspectiva, nosso estudo teve como objetivo investigar os efeitos da dieta hiperlipídica e do treinamento aeróbico em esteira no peso corporal, no consumo alimentar, na composição da massa corporal magra, nos lipídeos plasmáticos, no peso relativo do fígado e na aterosclerose em camundongos deficientes em apoE.

\section{MÉTODOS}

\section{Delineamento do estudo}

Foram utilizados 26 camundongos deficientes para o gene que expressa a apoE, com 12 semanas de vida (recém-desmamados). Esses foram distribuídos em quatro grupos: NS (dieta normolipídica e sedentário, $n=5$ ), HS (dieta hiperlipídica e sedentário, $n=5$ ), NT (dieta normolipídica e treinamento em esteira, $\mathrm{n}=8$ ) e HT (dieta hiperlipídica e treinamento em esteira, $n=8$ ).

Cada grupo foi alojado em gaiolas de polipropileno e mantido em ambiente com temperatura média de $21^{\circ} \mathrm{C}$, com alternância de período de 12 horas claro/escuro. Todos receberam água e dieta a vontade. 0 período do experimento foi de 12 semanas, e antes da eutanásia todos ficaram em jejum por 12 horas.

Após a eutanásia em ambiente com $\mathrm{CO}_{2}$, o sangue foi coletado por punção na região abdominal, sendo imediatamente centrifugado a 4.000 rotações por minuto (rpm) por 15 minutos. O soro foi congelado e mantido em freezer a $-20^{\circ} \mathrm{C}$ até a análise. Todas as vísceras foram retiradas, separando a carcaça para determinação da composição corporal da massa magra, o fígado para determinação de seu peso relativo e o coração junto com a aorta para análise das lesões ateroscleróticas.

Os camundongos foram procedentes do Biotério Central do Centro de Ciências Biológicas e da Saúde, da Universidade Federal de Viçosa. Os procedimentos empregados no estudo foram aprovados pelo Comitê de Ética do Departamento de Veterinária desta universidade (processo no. 13/2008), estando de acordo com o Colégio Brasileiro de Experimentação Animal (COBEA). A realização dessa pesquisa seguiu as resoluções específicas para experimentação animal (Lei n 6.638, de 08 de maio de 1979; e Decreto n² 24.645 de 10 de julho de 1934).

\section{Dietas}

A dieta normolipídica foi elaborada seguindo a proposta da AIN93 $\mathrm{M}^{8}$ e a dieta hiperlipídica baseando-se na proposta da Association of Official Analytical Chemistry - $\mathrm{AOAC}^{9}$, sendo confeccionadas manualmente e mantidas congeladas e protegidas da luz até o momento da utilização (tabela 1).

\section{Protocolo de atividade física}

Os camundongos dos grupos NT e HT foram submetidos a um programa progressivo de corrida na esteira (INSITH ${ }^{\circledR}$, Equipamentos Científicos, Ribeirão Preto - SP, Brasil), cinco dias por semana, 30 min/ dia, a uma velocidade de 15 metros por minuto (intensidade moderada segundo Meilhac et al. $\left.{ }^{10}\right)$, por 12 semanas consecutivas. A progressão da carga do exercício foi da seguinte forma: na $1^{\text {a }}$ e $2^{\text {a }}$ semana, os camundongos foram submetidos à treinamentos variados de exercício em esteira (10 a 30 minutos por seção/dia), 5 vezes por semana, à uma velocidade que variou de 10 a 15 metros por minutos para adaptação à atividade física proposta. Após essas duas semanas, as cobaias realizaram exercício com uma duração de 30 minutos por seção/dia, a uma velocidade média de 15 metros por minutos, 5 vezes por semana. 
Tabela 1. Composição das dietas experimentais ( $\mathrm{g} / \mathrm{kg}$ de dieta).

\begin{tabular}{|c|c|c|c|c|}
\hline Grupos/ingredientes & NS & NT & HS & HT \\
\hline Caseína & 140 & 140 & 200 & 200 \\
\hline Maltodextrina & 155 & 155 & - & - \\
\hline Sacarose & 100 & 100 & 500 & 500 \\
\hline Óleo de soja & 40 & 40 & 10 & 10 \\
\hline Celulose & 50 & 50 & 50 & 50 \\
\hline Mistura mineral & 35 & 35 & 50 & 50 \\
\hline Mistura vitamínica & 10 & 10 & 10 & 10 \\
\hline L-cistina & 1,8 & 1,8 & - & - \\
\hline Bitartarato de colina & 2,5 & 2,5 & 10 & 10 \\
\hline Colesterol & - & - & 10 & 10 \\
\hline Gordura hidrogenada & - & - & 150 & 150 \\
\hline Amido de milho & 465,692 & 465,692 & - & - \\
\hline Total (cal/kg dieta) & 4002,8 & 4002,8 & 4530 & 4530 \\
\hline \multicolumn{5}{|c|}{$\begin{array}{l}\text { Legenda: NS - dieta normolipídica e sedentário; NT - dieta normolipídica e treinamento em esteira; HS - dieta } \\
\text { hiperlipídica e sedentário; HT - dieta hiperlipídica e treinamento em esteira. Grupos NS e NT (dieta prepara- } \\
\text { da segundo AIN-93M, Reeves et al., 1997); grupos HS e HT (dieta preparada segundo a Association of Official } \\
\text { Analytical Chemistry, 1998). }\end{array}$} \\
\hline
\end{tabular}

No primeiro dia do experimento todos os animais foram pesados e distribuídos uniformemente em quatro grupos ( $P=0,97)$, sem diferença estatística no peso ponderal inicial. O peso corporal (PC) foi monitorado semanalmente até o último dia de experimento. Já o consumo alimentar (CA) foi verificado diariamente, sendo computado pela subtração das sobras da dieta do dia anterior. Tanto o PC quanto o CA foram mensurados utilizando uma balança semianalítica da marca Gehaka BG2000.

Para determinação da composição da massa corporal magra (CMCM) foi verificado o percentual de água (A\%), gordura (G\%) e proteína (P\%) das carcaças. Na análise do conteúdo de água, as carcaças vazias foram colocadas individualmente em pratos de alumínio e introduzidas num secador à temperatura de $105^{\circ} \mathrm{C}$ por 24 horas. A água da carcaça foi calculada pela diferença do peso da carcaça pré e pós-secagem. Após a secagem, as carcaças vazias foram maceradas e colocadas em cartuchos de papel filtro para a extração da gordura pelo método de Soxhlet ${ }^{11}$ durante oito horas, utilizando éter de petróleo como solvente. A gordura da carcaça foi determinada pela diferença do peso do cartucho contendo a carcaça pré e pós-desengordurada. A proteína foi calculada em triplicata pelo método indireto de determinação do nitrogênio (método de Kjeldahl) ${ }^{12}$, utilizando-se o fator 6,25 para conversão em proteína. As análises foram realizadas no Laboratório de Análise dos Alimentos do Departamento de Nutrição e Saúde da Universidade Federal de Viçosa.

\section{Lipídeos plasmáticos}

Para a análise dos lipídeos plasmáticos foram determinadas as concentrações do colesterol total (CT), lipoproteína de alta densidade $(\mathrm{HDL})$, triglicerídeos (TG), lipoproteína de baixa densidade (LDL) e lipoproteína de muito baixa densidade (VLDL). As determinações de CT, TG e HDL foram realizadas baseando-se no método enzimático colorimétrico ${ }^{13}$, utilizando kit enzimático da Bioclin ${ }^{\circledR}$ (Brasil) e analisador automático Cobas $^{\circledR}$ (Laboratório de Análises Clínicas da Divisão de Saúde da Universidade Federal de Viçosa, Brasil). A concentração de LDL e VLDL no plasma foi calculada baseando-se na fórmula de Friedewald ${ }^{14}$, a seguir: "LDL $=(C T-H D L)-(T G \times 0,20)^{\prime \prime}$ e "VLDL $=T G / 5$ ".

\section{Peso relativo do fígado}

O fígado extraído foi imerso, imediatamente, em solução fisiológica para retirar o excesso de sangue, em seguida foi secado e colocado sobre papel alumínio, pesado em balança digital, semianalítica da marca Gehaka BG2000. Após a aferição do peso, o fígado foi descartado. Para determinar o peso relativo do fígado (PRF) foi usada a seguinte relação: "PRF = (Peso do fígado/Peso corporal final) $\times 100 "$.

\section{Morfologia e quantificação das placas ateroscleróticas}

Após a eutanásia, o coração foi retirado em bloco com os vasos da base e a artéria aorta, sendo essa seccionada até sua porção torácica descendente. O coração foi seccionado transversalmente na base, tendo como referência macroscópica a válvula aórtica e fixado em paraformaldeído a 10\%. Os corações com as aortas foram fixados em parafina de forma orientada, de modo a obter cortes histológicos rigorosamente consecutivos medindo $5 \mu \mathrm{m}$ de espessura, a partir da superfície de inclusão, tendo sido obtidas, em geral, 10 lâminas por animal entre 5 um e 300 um distais da válvula aórtica, em que a lesão deu-se início e fim, respectivamente. Essas foram numeradas e coradas em hematoxilina e eosina (HE).

Os cortes histológicos da artéria aorta foram selecionados a partir da observação em microscópio óptico Zeiss acoplado a uma vídeo-câmera (Coolpix, Nikon, Düsseldorf, Alemanha), sendo as imagens congeladas, utilizando-se um programa de computador KS 400 (Laboratório de Morfometria, Departamento de Biologia Animal, Universidade Federal de Viçosa, Brasil) para obtenção da maior lesão aterosclerótica. A análise morfométrica dos cortes histológicos foi feita por um observador treinado, sem conhecimento prévio dos grupos analisados. Para análise da lesão aterosclerótica foi utilizado um programa de computador (Image Pro Plus ${ }^{\circledR}$ ), esse foi utilizado para medir a área real e área provável da aorta. A soma das áreas das lesões ateroscleróticas (local em que houve acúmulo lipídico) foi calculada pelo programa, sendo o resultado expresso em centímetros quadrados. Para garantir que não houvesse diferença entre os animais quanto ao tamanho total da aorta, esta área foi também mensurada, o que realmente foi confirmado ${ }^{15}$.

\section{Análises estatísticas}

Para verificar a influência dos fatores dieta e exercício físico sobre os valores de peso corporal inicial e final, consumo alimentar, composição da massa corporal magra, lipídeos plasmáticos, peso relativo do fígado e área total da lesão aterosclerótica, realizou-se a análise de variância a um fator (ANOVA). Quando a análise de variância apontou diferença estatisticamente significativa procedeu-se a comparação das médias pelo teste de Duncan. Os testes foram aplicados considerando-se um nível de significância de 5\% ( $<<0,05)$.

\section{RESULTADOS}

Peso corporal (PC), consumo alimentar (CA) e composição da massa corporal magra (CMCM)

O PC dos animais apresentou diferença durante o período experimental. Entretanto, ao final das 12 semanas, a média dos pesos não apresentou diferença significativa entre os grupos sedentários e exercitados, assim como entre os grupos que receberam dieta normolipídica e hiperlipídica $(P=0,18)$.

Ao analisar o CA, verificou-se diferença estatística significativa entre os grupos ( $P<0,001)$. O grupo HS demonstrou maior CA em relação aos grupos NS, NT e HT. Observou-se, ainda, que o grupo NT consumiu mais dieta que os grupos HT e NS.

Em relação a CMCM, não foram observadas alterações no percentual de gordura (G\%) e no percentual de proteína (P\%) nos animais treinados em relação aos seus respectivos controles $(P>0,05)$. Entretanto, o percentual de água (A\%) da carcaça foi significativamente maior nos indivíduos treinados, nos quais o grupo HT demonstrou maior A\% que os grupos HS e NS e o grupo NT, maior que o grupo HS ( $P=0,006)$.

Os efeitos dos lipídeos dietéticos e do exercício físico sobre o PC, CA e CMCM em camundongos apoE ${ }^{(-/)}$estão apresentados na tabela 3. 
Tabela 3. Peso corporal inicial e final (gramas), consumo alimentar (gramas) e composição da massa corporal magra (\%) em camundongos $\mathrm{ApoE}^{(--)}$.

\begin{tabular}{c|c|c|c|c}
\hline Grupos/variáveis & NS & HS & NT & HT \\
\hline Peso inicial & $19,95 \pm 3,59$ & $19,54 \pm 2,36$ & $20,35 \pm 3,36$ & $20,22 \pm 3,39$ \\
\hline Peso final & $23,36 \pm 2,48$ & $22,33 \pm 1,39$ & $23,51 \pm 2,33$ & $25,04 \pm 2,16$ \\
\hline Consumo alimentar & $340,16 \pm 6,32$ & $365,3 \pm 5,09 *$ & $350,4 \pm 7,81+$ & $336,68 \pm 6,23$ \\
\hline Água & $62,62 \pm 1,47$ & $61,71 \pm 1,62$ & $64,23 \pm 2,46 \neq$ & $65,62 \pm 1,49 \S$ \\
\hline Gordura & $8,01 \pm 2,54$ & $5,60 \pm 1,72$ & $6,52 \pm 2,84$ & $5,44 \pm 1,84$ \\
\hline Proteína & $19,44 \pm 1,91$ & $20,11 \pm 1,90$ & $18,96 \pm 1,02$ & $19,53 \pm 1,44$ \\
\hline
\end{tabular}

egenda: Valores estão expressos em médias \pm DP. ANOVA One Way e post-hoc de Duncan's Method. * $P<0,05$ comparando-se com o NS, NT e HT; $+\mathrm{P}<0,05$ comparando-se com HT e NS; $\neq \mathrm{P}<0,05$ comparando-se com o HS e NS; $\S \mathrm{P}<0,05$ comparando-se com o HS.

\section{Lipídeos plasmáticos}

Observou-se aumento significativo $(\mathrm{P}<0,001)$ dos valores absolutos de colesterol total (CT) no grupo HT em comparação com o grupo normolipídico com a mesma situação de exercício ou sedentário e também em relação ao grupo HS. Foi observado, também, valor significativamente maior de CT $(\mathrm{P}<0,001)$ do grupo hiperlipídico sedentário quando comparado com o grupo normolipídico treinado. Os níveis de triglicerídeos (TG) tiveram reduções significativas $(P<0,001)$ com o treinamento físico no grupo com dieta hiperlipídica em comparação com os grupos que se alimentaram com dieta normolipídica treinado e hiperlipídica sedentário.

Com relação ao teor de HDL, observou-se variação dos níveis entre os grupos, salientando aumento significativo no grupo HS em comparação com os grupos NT e HT ( $P=0,006)$. Observou-se, também, diferenças significativas nos níveis de VLDL entre os grupos $(P=0,001)$, salientando a redução dessa lipoproteína no grupo HT quando comparado com os demais grupos. Em relação ao LDL, foi verificado um aumento significativo no grupo HS em relação aos demais grupos e, também, uma alteração significativa entre os grupos HS e NT $(P<0,001)$. Os dados das análises estão apresentados na tabela 4.

Tabela 4. Níveis de lipídeos plasmáticos (mg/dL) em camundongos ApoE $E^{(-/)}$.

\begin{tabular}{|c|c|c|c|c|}
\hline $\begin{array}{l}\text { Grupos/ } \\
\text { variáveis }\end{array}$ & NS & HS & NT & HT \\
\hline Colesterol total & $367,60 \pm 88,12$ & $503,00 \pm 115,80 \dagger$ & $320,87 \pm 157,81$ & $858,75 \pm 140,84$ * \\
\hline TG & $63,00 \pm 15,19$ & $66,00 \pm 21,88$ & $72,00 \pm 18,14$ & $22,75 \pm 12,36 \neq$ \\
\hline $\mathrm{HDL}-\mathrm{C}$ & $25,40 \pm 3,64$ & $37,60 \pm 13,12 \S$ & $23,81 \pm 11,81$ & $14,83 \pm 3,95$ \\
\hline VLDL-C & $12,60 \pm 2,71$ & $13,20 \pm 4,37$ & $14,40 \pm 3,62$ & $4,55 \pm 2,47 / /$ \\
\hline LDL-C & $329,60 \pm 77,54$ & $452,20 \pm 114,63 \#$ & $282,66 \pm 166,92$ & $839,36 \pm 139,94$ \\
\hline
\end{tabular}

\section{Peso relativo do fígado (PRF)}

Os fígados dos diferentes grupos não apresentaram PRF semelhantes (figura 1). Devendo ser ressaltado o aumento significativo do PRF no grupo HT em comparação com os grupos NT e NS e do grupo HS em relação ao grupo NT $(P=0,005)$.

\section{Morfologia e quantificação das placas}

Com o intuito de avaliar os efeitos da dieta hiperlipídica e do treinamento aeróbico em esteira na formação da lesão aterosclerótica, o aspecto histológico e a morfometria da aorta foram estudados. Sendo assim, observou-se capa fibrosa, cristais de colesterol, macrófagos xan- tomizados e núcleos de células musculares lisas em migração nas lesões em todos os grupos (NS, HS, NT e HT). Porém, no grupo HT (figura 2, letra D) observou-se grande extensão da parede arterial espessada por deposição de massa difusa de material necrótico, contendo cristais de colesterol, recoberta por células xantomizadas, células musculares lisas e células inflamatórias que constituíram a capa fibrosa $\left(^{*}\right)$. Já no grupo NT (figura 2, letra C), foi observada uma pequena proporção da área espessada da parede ocupada por alterações degenerativo-necróticas e grande quantidade de cristais de colesterol (†), ao contrário da lesão do grupo HS (figura 2, letra B), que apresentou grande quantidade de células necróticas degenerativas e pouca presença de cristais de colesterol (†). Tanto no grupo NS quanto no grupo HS (figura 2, letras A e B), nota-se alteração da polaridade celular da camada muscular íntima, com evidência de atividade sintética e proliferativa dos núcleos, sendo esses vesiculosos e com nucléolos evidentes, além de início de um processo de mitose. Observou-se, ainda, presença de algumas células com citoplasma vacuolizado na íntima e núcleos de células musculares lisas aumentados de volume (§).

Em relação à área da lesão, os animais treinados demonstraram menores valores do que os sedentários, independente do tipo de dieta. Porém, somente no grupo HT a lesão foi significativamente menor $(P=0,025)$ que os grupos HS e NS (figura 3).

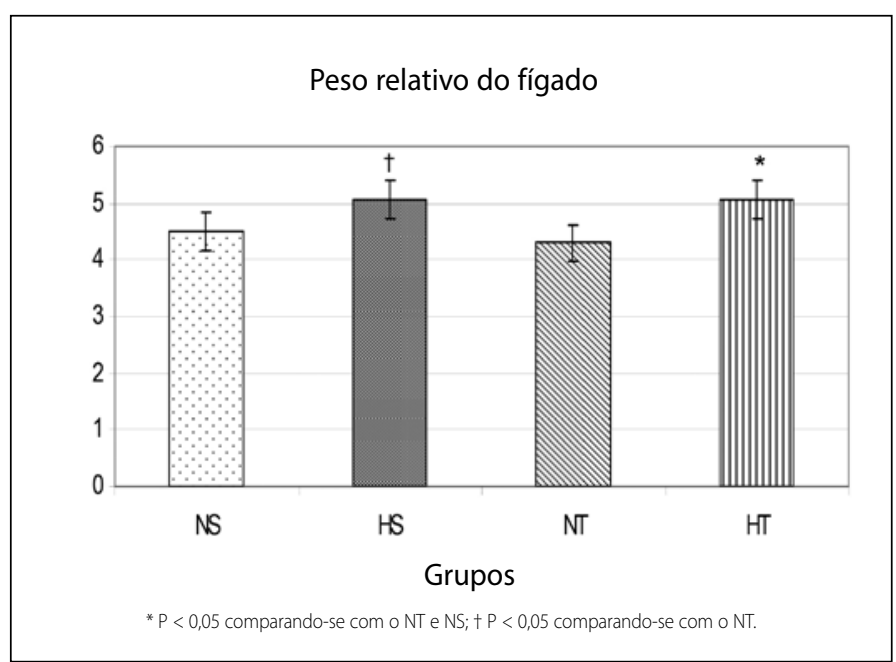

Figura 1. Valores estão expressos em médias \pm DP. Anova One Way e post-hoc de Duncan's Method.

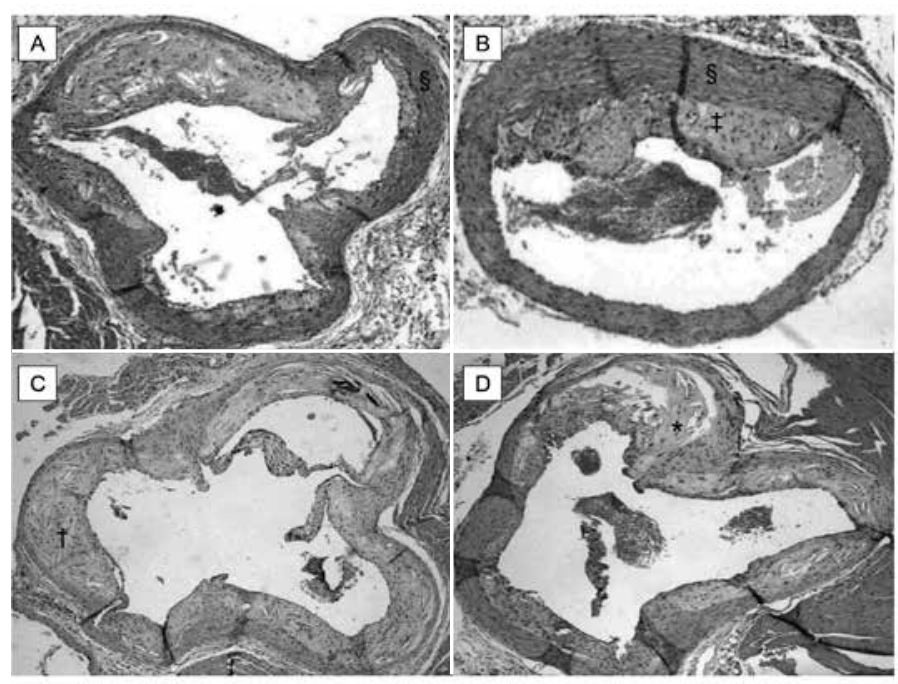

Figura 2. Letra "A" representa seção histológica do grupo NS; letra "B" representa seção histológica do grupo HS; letra "C" representa seção histológica do grupo NT; e letra" $\mathrm{D}$ " representa seção histológica do grupo HT. 


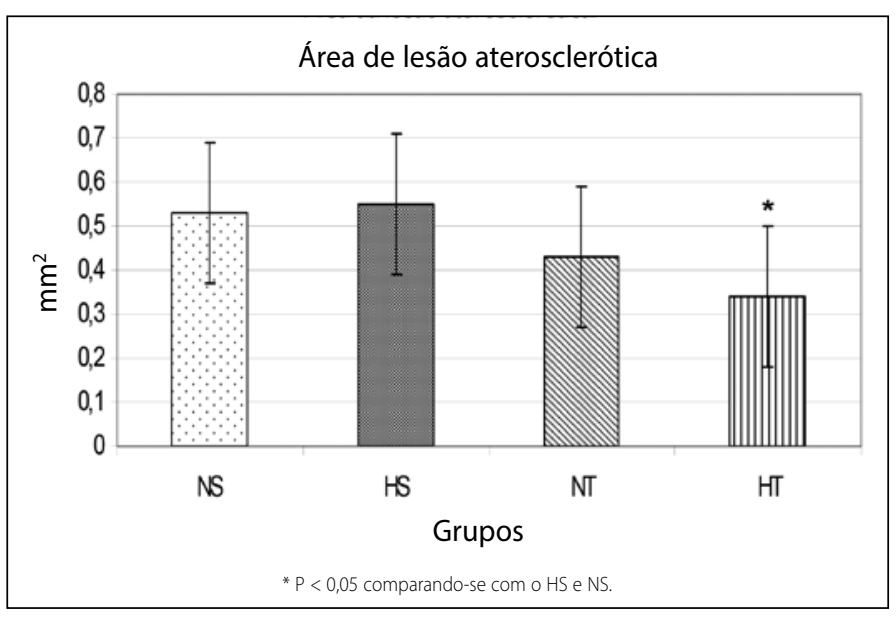

Figura 3. Valores estão expressos em médias \pm DP. Anova One Way e post-hoc de Duncan's Method.

\section{DISCUSSÃO}

No presente estudo, o PCF dos animais dos grupos analisados não mostrou diferenças significativas. Frente a esse resultado, pode-se supor que a diferença na quantidade de calorias das duas dietas utilizadas, as quais apresentavam 4,0 kcal/g (grupos NS e NT) e 4,5 kcal/g (grupos HS e $H T$ ), não foi suficiente para induzir um aumento de peso nos animais. Os percentuais de gordura utilizados em relação ao valor calórico total das dietas, que foram de 4\% na dieta normolipídica e 25\% na dieta hiperlipídica, também não apresentaram influência sobre o ganho de peso dos animais, ainda que tenha sido acrescido à dieta do grupo hiperlipídico o dobro da necessidade de gordura recomendada para a espécie ${ }^{8}$.

O treinamento promoveu diminuição do CA somente para os animais que receberam dieta hiperlipídica quando comparados com seu controle, o grupo HS. A redução no CA sugere aumento da saciedade ocasionada pela elevação nos níveis de substratos metabólicos plasmáticos como glicose, triglicerídeos e colesterol ${ }^{16}$. Contudo, em animais alimentados com dieta normolipídica, esse fenômeno não ocorreu, pois o treinamento induziu um maior consumo de dieta comparando-se com o grupo sedentário (NS). Esse fato pode ser explicado, possivelmente, pela maximização da utilização de carboidratos oriundos da dieta e do próprio organismo, através da depleção do glicogênio hepático e muscular causada pelo exercício e, com isso, causando um aumento no $\mathrm{CA}^{17}$. Por esse motivo, a adoção da suplementação lipídica em associação ao treinamento físico tem sido sugerida, visando maximizar a utilização deste tipo de substrato em detrimento aos estoques de carboidrato. Esse fato promoveria, assim, um efeito poupador de glicogênio com melhora do desempenho e atraso na instalação da fadiga ${ }^{7}$. Porém, estes mesmos dois fatores atuam de maneira oposta com relação ao metabolismo de carboidratos. O treinamento físico aumenta o conteúdo de glicogênio estocado enquanto que a dieta hiperlipídica tem sido associada com diminuição na taxa de glicólise e na síntese de glicogênio ${ }^{18}$.

Em relação ao aumento do A\% nos grupos exercitados, sugere-se que o treinamento preservou a massa corporal magra (MCM), como pode ser constatada pela meta-análise realizada por Garrow e Summerbell ${ }^{19}$. Os autores sugeriram que esse efeito ocorre, em parte, pela preservação do seu componente fluídico, formado por água e glicogênio, pois indivíduos exercitados apresentaram aumento dos estoques de glicogênio muscular e da água intracelular associada a este composto (1 grama de glicogênio para 2 a 4 gramas de água ${ }^{20}$. Esse fato é observado na análise química do $\mathrm{A} \%$ de nosso estudo, pois os grupos treinados demonstraram maior concentração desse fluido na carcaça em comparação com sedentários $(P<0,05)$. Deste modo, concluiu-se que parte da preservação da $M C M$, resultante do treinamento, poderia ser decorrente do aumento do volume das células musculares e não da conservação das proteínas estruturais que as compõem, pois em relação ao percentual de proteína da carcaça os animais não tiveram diferenças significativas.

Durante o exercício, os ácidos graxos livres (AGLs) são mobilizados do tecido adiposo e transportados pelos vasos sanguíneos até o músculo, a fim de servir como substrato energético; como consequência, eles aumentam suas concentrações no sangue ${ }^{21}$. Em relação ao metabolismo dos lipídeos plasmáticos, o treinamento associado à dieta hiperlipídica aumentou a concentração de $\mathrm{CT}$, pois os animais do grupo HT demonstram maiores valores do que os indivíduos dos grupos NT, HS e NS. De fato, estudos anteriores evidenciaram que a constante utilização de gordura como substrato energético pode acelerar a biossíntese de colesterol ${ }^{22}$. Contudo, a dieta hiperlipídica nos animais treinados aumentou a utilização dos TG e VLDL, pois o grupo HT demonstrou menores valores em comparação com os grupos NT, HS e NS. Curi et al. ${ }^{23}$ afirmaram que a insaturação da cadeia carbônica (saturada e insaturada) influencia diretamente o aumento da disponibilidade dos AGLs provenientes dos TG do tecido adiposo, dos quilomícrons (QM) e VLDL circulantes e do próprio tecido muscular, fato esse potencializado pelo treinamento aeróbico. Em exercícios aeróbicos ocorre acentuada melhora de fatores que modulam o fluxo e a capacidade oxidativa dos músculos esqueléticos: capilarização, lipólise dos TG no tecido adiposo e circulante, transporte dos AGLs do sangue ao sarcoplasma, disponibilidade e taxa de hidrólise dos TG intramusculares, transporte dos AGLs pela membrana mitocondrial, ativação das enzimas da cadeia oxidativa e adaptações hormonais, especialmente da insulina e das catecolaminas ${ }^{24}$.

A maior concentração de HDL no sangue está relacionada ao maior conteúdo de gordura da dieta ${ }^{25}$; porém, quando ocorre uma interação entre dieta hiperlipídica e exercício, o mesmo fenômeno não acontece, fato evidenciado em nosso estudo. Isso pode ser explicado pelo fato de os camundongos serem deficientes em apoE, pois esta apolipoproteína (apoLP) faz parte da estrutura da HDL e está envolvida indiretamente na síntese de novas moléculas de HDL. Além de a apoE possuir a função de reconhecimento dos remanescentes de quilomícrons (QMr) e pelos receptores celulares específicos (B e E) no fígado ${ }^{26}$. No entanto, quando QM e VLDL perdem TG, ocorre uma diminuição do volume da partícula e, desta forma, componentes de superfície como colesterol livre (CL), fosfolipídeos (FP) e apoLP são liberados, formando macroagregados moleculares de forma discoidal, sendo esses precursores da HDL plasmáti$\mathrm{ca}^{27}$. Sendo assim, como o animal é deficiente em apoE, a remodelação intravascular de LP ricas em TG (QM e VLDL) não ocorre. Por outro lado, o exercício aumenta a atividade da lípase lipoproteica (LLP), cuja função é elevar o metabolismo dos QM e VLDL. Desta forma, componentes de superfície (CL, FP e apoLP) são liberados para formação de HDL, porém em indivíduos apo $E^{(--)}$esse mecanismo fica comprometido, pois o transporte de colesterol esterificado para os QM é interrompido e, com isso, novas moléculas de HDL também não serão formadas ${ }^{26}$. É sabido que o exercício realizado de maneira regular tem promovido aumento da HDL em humanos ${ }^{28}$, porém, não foi encontrado nenhum estudo que analisou os efeitos do exercício nesta LP em indivíduos deficientes em apoE ou, até mesmo, estudos com associação entre polimorfismo da apoE e HDL.

Neste estudo, também, foram analisadas as concentrações de LDL e foi constatado que os grupos com dieta hiperlipídica tiveram maiores valores ( $P$ $<0,001)$ dessa LP, independente se eram treinados ou sedentários. Sabe-se que a apoE possui ação no metabolismo das lipoproteínas, desempenhando um papel chave no transporte da molécula de colesterol, principalmente como ligante do receptor de LDL ${ }^{5}$. Desta forma, sugere-se que, em indivíduos deficientes na apoE ou com polimorfismo dessa apoLP, a dieta por si só possa aumentar as concentrações de LDL no sangue e, associada ao exercício, esse efeito possa ser potencializado, pois o pool dessa LP pode ficar elevado pelo 
aumento da atividade da lípase hepática (LH). Essa enzima tem ação sobre as lipoproteínas de densidades intermediárias (IDL) transformando-as em partículas menores e mais densas, que são as LDL ${ }^{29}$.

Quanto às alterações no peso do fígado, foi observado um aumento no PRF $(P<0,05)$ nos animais submetidos a dieta hiperlipídica, independente do treinamento. $O$ fígado é o principal regulador do fluxo metabólico corporal, através do sistema porta-hepático da circulação geral, através da artéria hepática e do sistema linfático. A capacidade de captação, especialmente de lipídios, pelos hepatócitos pode ser a principal explicação para o aumento do PRF e, com isso, podendo resultar em esteatose hepática ${ }^{30}$. O aumento na deposição de gordura no fígado pode ser também ocasionado devido ao aumento na disponibilidade de AGLs, com concomitante ausência de aumento na $\beta$-oxidação ${ }^{31}$. Outro fato que pode ser levado em consideração é a possibilidade de um controle endócrino gerado pelos animais alimentados com dieta rica em lipídeos, gerando maior produção hepática de gorduras e menor utilização destas com consequente acúmulo hepático ${ }^{32}$. Nesse sentido, sugere-se que o aumento do PRF encontrado em nosso estudo pode ser devido a uma maior quantidade de gordura depositada nele, pois em estudos que analisaram o efeito da dieta hiperlipídica, foi verificado um maior acúmulo de gordura no fígado com consequente presença de esteatose hepática ${ }^{33}$.

Por fim, ao analisar a área da lesão aterosclerótica, encontramos um resultado surpreendente, pois os animais treinados submetidos à dieta hiperlipídica (HT) demonstraram menores valores na área da lesão quando comparados com os animais sedentários, independente do tipo da dieta $(P<0,05)$. Esse fato nos chamou a atenção, pois o grupo HT teve valores significativamente maiores no $C T$ e LDL, menor valor na HDL e maior PRF. Com isso, sugere-se que o treinamento pode ter influenciado a formação e progressão da placa, pois estudos demonstraram que o exercício físico aeróbico melhora os sistemas de defesa orgânicos contra

\section{REFERÊNCIAS}

1. Van Gaal LF, Mertens IL, De Block CE. Mechanisms linking obesity with cardiovascular diseases. Nature 2006; $444: 875-80$

2. Teodoro GB, Natali AJ, Fernades SAT, Peluzio MCG. A influência da intensidade do exercício físico aeróbio no processo aterosclerótico. Rev Bras Med Esporte 2010;16:382-7.

3. Campos W, Neto AS, Bozza R, Ulbrich AZ, Bertin RL, Mascarenhas LPG, et al. Atividade física, consumo de lipídios e fatores de risco para aterosclerose em adolescentes. Arq Bras Cardiol 2010;94:601-7.

4. Guedes DP, Guedes JERP, Barbosa DS, Oliveira JA, Stanganelli LCR. Fatores de risco cardiovasculares em adolescentes: indicadores biológicos e comportamentais. Arq Bras Cardiol 2006;86:439-50,

5. Schwanke CHA, Cruz IBM, Leal, NF, Scheibe R, Moriguchi Y, Moriguchi EH. Análise da associação entre polimorfismo do gene da apolipoproteína E e fatores de risco cardiovasculares em idosos longevos. Arq Bras Cardiol 2002;78:561-70.

6. Brandão AC, Pinheiro Junior S, Pinhel MA, Anacleto AM, De Godoys JMP, De Godoy MF, et al. Polimorfismo genético da apolipoproteína E na doença arterial periférica. J Vasc Br 2004;3:317-22.

7. Bernardes D, Manzoni MSJ, Souza CP, Tenório N, Damaso AR. Efeitos da dieta hiperlipídica e do treinamento de natação sobre o metabolismo de recuperação ao exercício em ratos. Rev Bras Educ Fís Esp 2004;18:191-200

8. Reeves PG. Components of the AIN-93 diets as improvements in the AIN-76A diet. J Nutr 1997;127 (supl. 5):838-41.

9. Association of Official Analytical Chemists. Official methods of analysis of AOAC 16th ed. International. Washington (DC): AOAC International; 1998.

10. Meilhac O, Ramachandran S, Chiang K, Santanam N, Parthasarathy S. Role of arterial wall antioxidant defense in beneficial effects of exercise on atherosclerosis in mice. Arterioscler Thromb Vasc Biol 2001;21:1681-8.

11. Cecchi HM. Fundamentos teóricos e práticos em análise de alimentos. Campinas: Unicamp; 1999.

12. Cannon DC, Olitzky I, Inkpen YA. Proteins. In: Henry RJ, Cannon DC, Winkelman JW, editors. Clinica chemistry: principles and techniques. 2th ed. Hargerstown: Haper \& How 1974;1:405-502.

13. Fossati $P$, Prencipe L. Serum triglycerides determined colorimetrically with an enzyme that produces hydrogen peroxide. Clinic Chem 1982;28:2077-80

14. Friedewald WT, Levy RI, Fredrickson DS. Estimation of the concentration of low-density lipoprotein cholesterol in plasma, without use of the preparative ultracentrifuge. Clin Chem 1972;18:499-502.

15. Paigen B, Morrow A, Holmes PA, Mitchell D, Williams RA. Quantitative assessment of atherosclerotic lesions in mice. Atherosclerosis 1987;68:231-40.

16. Himaya A, Fantino M, Antoine JM, Brondel L, Louissylvestre A. Satiety power of dietary fat: a new appraisal. Am J Clin Nutr 1997;5:1410-8.

17. Aoki MS, Seelaender MCL. Suplementação lipídica para atividades de endurance. Rev Paul Ed Fis 1999;13:230-8

18. Kim CH, Youn JH, Park JY, Hong SK, Park KS, Park SW, et al. Effects of highfat diet and exercise training on intracellular glucose metabolism in rats. Am J Physiol 2000;278:977-84.

19. Garrow JS, Summerbell CD. Meta-analisis: effect of exercise, with or without dieting, on the body composition of overweight subjects. Eur J Clin Nutr 1995;49:1-10.

20. Heymsfield SB, Casper K, Hearn J, Guy D. Rate of weight loss. During underfeeding: relation to level of physical activity. Metabolism 1989;38:215-23.

21. St Clair Gibson A, Schabort EJ, Noakes TD. Reduced neuromuscular activity and force generation during prologed cycling. Am J Physiol Regul Integr Comp Physiol 2001;281:187-96. aterosclerose, diminuindo o estresse oxidativo e aumentando a síntese de enzimas antioxidantes, aumentando a vasodilatação dos vasos sanguíneos via óxido nítrico (NO) e óxido nítrico sintase endotelial (eNOS) e diminuindo a inflamação sistêmica pela diminuição da produção de citocinas pró-inflamatórias e aumento de fatores anti-inflamatórios ${ }^{34-36}$.

Estudos têm sido feitos para a comprovação dos efeitos benéficos do exercício físico aeróbico na redução e prevenção de doenças cardiovasculares (DC) 2,34,37. Assim, a Sociedade Brasileira de Cardiologia ${ }^{38}$ recomenda a prática regular de exercício aeróbico, em uma frequência semanal de três a seis vezes, com duração de 30 a 60 minutos por sessão e em intensidade moderada (40 a 60\% da frequência cardíaca máxima) para prevenção e tratamento da aterosclerose e dislipidemias.

\section{CONCLUSÃO}

O treinamento aeróbico de intensidade moderada, combinado ou não com dieta hiperlipídica, reduz lesões ateroscleróticas em camundongos apo ${ }^{(--)}$com aterosclerose preexistente. Entretanto, esta redução da lesão parece não ser dependente da apoE, e é independente das mudanças do colesterol no plasma. Em pessoas com deficiência da apoE, a redução do colesterol e o aumento do treinamento físico podem atenuar significativamente a formação da lesão aterosclerótica ao longo das mudanças na formação do indivíduo.

\section{AGRADECIMENTOS}

À Universidade Federal de Viçosa, especialmente, aos Departamentos de Nutrição, Educação Física, Biologia e Veterinária pela colaboração nas análises dos materiais. À CAPES, pela concessão da bolsa de pesquisador.

Todos os autores declararam não haver qualquer potencial conflito de interesses referente a este artigo.

22. Hanson DL, Lorenzen JA, Morris AE, Ahrens RA. Effects on fat intake and exercise on serum cholestero and body composition of rats. Am J Physiol 1967;213:347-52.

23. Curi R, Lagranha CJ, Rodrigues Jr JG, Pithon-Curi TC, Lancha Jr AH, Pellegrinotti IL, et al. Ciclo de Krebs como um fator limitante na utilização de ácidos graxos durante o exercício aeróbico. Arq Bras Endocrinol Metab 2003;47:135-43

24. Horowitz JF, Klein S. Lipid metabolism during endurance exercise. Am J Clin Nutr 2000;72(supl 2):558-63.

25. Katan MB. Effect of low-fat diets on plasma high-density lipoprotein concentrations. Am J Clin Nutr 1998;68:573-6.

26. Nunes APOB, Vinagre, CGCM, Maranhão RC. Exercício físico e metabolismo de lípides plasmáticos. Em: Negrão CE, Barretto ACP, editores. Cardiologia do exercício: do atleta ao cardioplata. Barueri: Manole; 2006.

27. Garcia RC, Oliveira HCF. Fisiologia da lipoproteínas. Em: Quintão ECR, editor. Colesterol e aterosclerose Rio de Janeiro: Qualitymark; 1992.

28. Couillard C, Despres JP, Lamarche B, Bergeron B, Gagnon J, Leon AS, et al. Effects of endurance exercise training on plasma $\mathrm{HDL}$ cholesterol level depend on levels of triglycerides. Arterioscler Thromb Vasc Biol 2001;21:1226-32.

29. Brown MS, Goldstein JL. A receptor-mediated pathway for cholesterol homeostasis. Science 1986;232:34-47.

30. Bradbury MW. Lipid metabolism and liver inflammation. I. Hepatic fatty acid uptake: possible role in steatosis. Am J Physiol Gastrointest Liver Physiol 2006;290:194-8.

31. Gauthier MS, Couturier K, Latour JG, Lavoie JM. Concurrent exercise prevents high-fat-diet-induced macrovesicular hepatic steatosis. J Appl Physiol 2003;94:2127-34

32. Osumi Y, Nagasaka Y, Shimamoto K. Lipid metabolism in the rats with fatty liver caused by low protein diet and effects of the oral administration of L-methionine, $\mathrm{L}$-cysteine, pantethine and calcium pantothenate upon it. Jpn J Pharmacol 1969;19:74-88.

33. Zambon L, Duarte FO, Freitas LC, Scarmagnani FRR, Damaso A, Duarte ACGO, Sene-Fiorese M. Efeitos de dois tipos de treinamento de natação sobre a adiposidade e o perfil lipídico de ratos obesos exógenos. Rev Nutr 2009;22:707-15.

34. Napoli C, Ignarro SW, de Nigris F, Lerman LO, Rossi L, Guarino C, et al. Long-term combined beneficia effects of physical training and metabolic treatment on atherosclerosis in hypercholesterolemic mice. PNAS 2004;101:8797-802.

35. De Moraes C, Davel APC, Rossoni LV, Antunes E, Zanesco A. Exercise training improves relaxation response and SOD-1 expression in aortic and mesenteric rings from high caloric diet-fed rats. BMC Physiology 2008:8:12

36. Czarkowska-Paczek B, Zendzian-Piotrowska M, Bartlomiejczyk I, Przybylski J, Gorski J. The effect of acute and prolonged endurance on transformimg cgrwth factor- $\beta$ generation in rat skeletal and heart muscle. J Phisyol Phamacol 2009;60(Supl 4):157-62.

37. Maeda S, Tanabe T, Miyauchi T, Otsuki T, Sugawara J, lemitsu M, et al. Aerobic exercise training reduces plasma endothelin-1 concentration in older women. J Appl Physiol 2003;95:336-41.

38. Sociedade Brasileira de Cardiologia. IV Diretriz Brasileira Sobre Dislipidemias e Prevenção da Aterosclerose Departamento de Aterosclerose da SBC. Arq Bras Cardiol 2007:88(supl. 1):2-19. 